\title{
Study of lipoprotein (a) in patients of acute coronary syndrome and effect of low dose nicotinic acid on its serum level
}

Rajib Rajbhandari,* Roshan Raut, Sujeeb Rajbhandari, Rabi Malla, Arun Maskey, Man Bahadur KC, Yubraj Limbu, Yadab Bhatta, Deewakar Sharma, Ranjit Sharma, Sajan Baidhya, Chandra Mani Adhikari, Dipankar Prajapati

\author{
Accepted on \\ July $6^{\text {th }}, 2013$

\section{DOI Name} \\ http://dx.doi.org/10.3126/jaim.v2i2.8778

\section{Keywords} \\ lipoprotein(a), acute coronary syndrome, \\ nicotinic acid

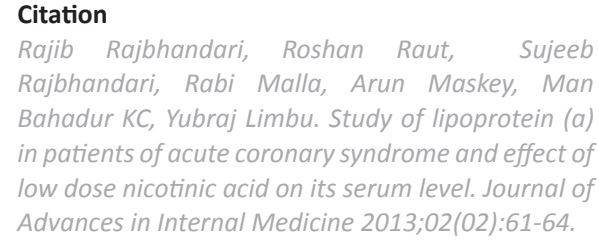

\begin{abstract}
Background and aims - Lipoprotein(a) is not an established independent risk factor. This study aims to observe whether lipoproteina(Lpa) is significantly high in patients of acute coronary syndrome (ACS) in comparison to healthy control. The second part of the study is aimed to know whether oral nicotinic acid treatment lowers Lpa level significantly.

Methods - This is a prospective case control study with quasi-experimental design done on acute coronary cases admitted in our centre. Total 200 cases of ACS were included as cases and 46 healthy volunteers were controls. In later part of the study those patients who had high Lpa and were eligible were treated with nicotinic acid for 3 months and Lpa was measured again.

Results - Mean Lp(a) level was $39.7 \mathrm{mg} / \mathrm{dl}( \pm 17)$ among the 200 ACS cases admitted in CCU with the maximum of 83 and minimum of 14 . Among controls mean Lpa was $22 \mathrm{mg} / \mathrm{dl}( \pm 3.04)$ with maximum of 44 and minimum of $17 \mathrm{mg} / \mathrm{dl}$. Difference was significant with $p$ value of $<0.01$. It was observed that nobody discontinued the drug because of side-effects. In these patients there was significant reduction in serum Lpa level from $74.1 \mathrm{mg} / \mathrm{dl}$ ( \pm SD22.2) to $43.2 \mathrm{mg} /$ $\mathrm{dl}( \pm$ SD21.9) $\mathrm{p}<0.01$.

Conclusion - The serum level of Lpa was significantly high in patients with ACS patients in comparison to control. This difference however was not significant between STEMI and NSTEMI patients. Low dose Nicotinic acid reduces Lpa significantly and is well tolerated by the patients.
\end{abstract}

\section{INTRODUCTION}

The conventional risk factors for coronary artery disease are dyslipidemia cigarette smoking obesity old age male sex etc. However, there are other risk factors which are strongly associated with the coronary artery disease but do not have strong evidence, such as Lpa, serum fibrinogen level etc. Though many studies have yielded a positive association between Lipoprotein (a) $\{\mathrm{Lpa}\}$ and Coronary Artery disease (CAD), many studies have shown controversial data.

Lpa consists of an LDL-like particle and the specific apolipoprotein(a) [apo(a)], which is covalently bound to the apoB of the LDL like particle. Lp(a) plasma concentrations are heritable and primarily controlled by the apolipoprotein(a) gene [LPA] . The structure of Ipa is similar to plasminogen and TPA (tissue plasminogen activator). It competes with plasminogen for its binding site, leading to reduced fibrinolysis. Lpa transports the more atherogenic proinflammatory oxidized phospolipids which attract inflammatory cells to vessel walls, and leads to smooth muscle cell proliferation. Lpa carries cholesterol and thus contributes to atherosclerosis. High Lpa predicts risk of early atherosclerosis independently of other cardiac risk factors. Serum Lpa level may be affected by kidney failure, but are only minimally affected by diet, exercise, and

\footnotetext{
* Corresponding author Dr. Rajib Rajbhandari Department of cardiology, Shahid Gangalal National Heart center, Kathmandu, Nepal Email: rajibrbhandari@yahoo.com
} 
other environmental factors. Most commonly prescribed lipidreducing drugs have insignificant effect on Lpa concentration.

High Lpa predicts risk of early atherosclerosis independently of other cardiac risk factors, including low density lipoprotein(LDL). Lpa has not been established as a strong independent risk factor in many studies from North America ${ }^{1}$ and Europe. ${ }^{2,3}$ However in some study Ipa is considered important risk factor in South Asians settled abroad. ${ }^{4}$

Some ethnic groups are known to have higher Ipa levels than others which has been attributed to the genetic heterogeneity between different ethnic groups. Although most of the studies consider Lpa as emerging coronary artery risk factor many studies hesitate to take it as independent risk factor. So it is not yet certain whether Lpa is an independent risk factor for coronary artery disease in South Asian population.The only accepted therapy for significantly elevated Lpa levels today is LDL apheresis, which is invasive, expensive and not available in many centers. ${ }^{5}$

Since it will take some time until these approaches are available for clinical use, the only widely applicable and safe pharmacological agents to lower Lpa levels are nicotinic acid. Nicotinic acid has been shown to be safe and it lowers Lpa levels by up to $30 \%$ in several controlled studies, however the exact quantity of lowering and the mechanism is not clear ${ }^{6-8}$ and also the apprehension of side-effects in high doses are quite bothersome.

Nicotinic acid reduces the Lpa but in high doses (more than $2 \mathrm{gram} / \mathrm{d}$ ) which is usually not tolerated by the patients. There are not many randomized control trials using low dose nicotinic acid to reduce Lpa. If these ACS with high Lpa are treated, incidents of ACS may decrease.

The aim of the study was to observe whether serum Ipa level is significantly high in patients of acute coronary syndrome (ACS) in comparison to controls, to find out if there are any differences in Lpa level between different types of acute coronary syndrome and to observe whether treatment with oral low dose nicotinic acid lowers the serum Lpa level.

\section{METHODS:}

The study was conducted in Shahid Gangalal National Heart centre (SGNHC) Kathmandu and it was a quasi-experimental study design. Total two hundred patients admitted in the one year were included as cases in the study. This is a prospective study started from October 2008 and continued for about 2 years. The volunteers attending SGNHC OPD patients who came for comprehensive cardiac check-up were taken randomly as controls. Their risk factors were matched with the acute cases admitted as ACS. All patients with ACS admitted in CCU of SGNHC served as cases for the study and treatment with nicotinic acid. Informed Consent was taken from every volunteer and patient before the sample was withdrawn; approval was obtained from hospital ethics committee for the study.

Ten milliliter of blood was drawn in the morning and was sent immediately to lab for measurement of Lpa in ACS cases admitted to CCU. Sample from the control was taken from out patient door (OPD) cases. Measurement was done by turbidometry method. The next part of the study was to administer oral nicotinic acid to those patients who had high level of Lpa.

Inclusion criteria: All patients with age between30-65 attending SGNHC OPD who volunteered for the study, served as control; all patients admitted to CCU as acute coronary syndrome served as cases. And all patients with ACS having high Lpa level were enrolled for the treatment with nicotinic acid in the later part of the study.

Exclusion criteria: Patients with deranged renal function (which affects the Lpa level) and uncontrolled diabetics and those in killip class 2 or more were excluded from the study. Also those who underwent percutaneous coronary intervention (PCI) were also excluded.

In the other part of the study, out of 200 ACS patients admitted in $\mathrm{CCU}, 24$ patients were selected for the treatment because of their high serum Lpa level; of these 20 were available for follow-up. Those who had increased level of Lpa ( $\geq 40 \mathrm{mg} / \mathrm{dl}$ ) were administered sustained- release nicotinic acid $375 \mathrm{mg}$ twice daily for 6weeks followed by $375 \mathrm{mg}$ thrice daily for another 6 weeks. Serum level of Lpa was checked after that. The other drugs were continued as before. Patients were enquired about the possible side-effects. All patients were on atorvastatin, and only two were on ezetimibe and one on fibrates and no one were on any other medicines like antiinflammatory or other medications like ayurvedic medicines.

Serum levels of control group and cases were measured and mean values calculated and expressed as mean \pm standard deviation. The serum levels between control group and acute coronary syndrome cases were compared. Also comparison was studied between unstable angina and $\mathrm{Ml}$ patients. The difference between two groups was calculated using or $z$ test or student $t$ test, whichever applicable using SPSS version 11.5. The $p$ value $<0.05$ was considered as significant.

\section{RESULTS}

In the first part of the study, among 200 ACS patients included in the study, their age ranged from minimum of 28 years to maximum of 86 years, with mean of 53.7years $( \pm 14.5)$. Male 
patients consisted of $86 \%$ among cases and $89 \%$ among controls. Among the cases $22 \%$ were unstable patients $16 \%$ were non-ST elevation myocardial infarction (NSTEMI) and $62 \%$ were ST elevation myocardial infarction (STEMI).

Among controls $55 \%$ smoked and $70 \%$ of the cases smoked cigarette. Hypertension was present among $30 \%$ of the controls and 50\% among cases. Non-insulin dependent diabetes mellitus (NIDDM) was present among $8 \%$ of the controls and $18 \%$ among the cases. Family history of premature coronary artery disease was present among present in $4 \%$ of the cases and $2 \%$ among the controls. Dyslipidemia was present among $12 \%$ of the controls and $30 \%$ of the cases.

Among the cases mean lipoprotein (a) level was $39.7 \mathrm{mg} / \mathrm{dl}$ $( \pm 17)$ with the maximum of 83 and minimum of 14 . Among the controls mean lipoprotein (a) was $22 \mathrm{mg} / \mathrm{dl}( \pm 5.04)$ with maximum of $44 \mathrm{mg} / \mathrm{dl}$ and minimum of $17 \mathrm{mg} / \mathrm{dl}$. Difference was significant with $p$ value of $<0.01$. The difference between STEMI and NSTEMI was not statistically significant with $p$ value of 0.78 . The difference between UA and MI was not statistically significant with $p$ value of 0.35 .

In the second part of the study that is the treatment with niacin, it was observed that nobody discontinued the drug because of the side-effects. There was a significant reduction in serum Lpa level from $74.1 \mathrm{mg} / \mathrm{dl}( \pm$ SD22.2) among $20 \mathrm{ACS}$ patients to $43.2 \mathrm{mg} / \mathrm{dl}( \pm S D 21.9) p<0.01$. The clinical events could not be studied because of inadequate follow-up and small sample size.

\section{DISCUSSION}

Analyzing characteristics of cases and controls, few things to be noted are the prevalence of different risk factors among controls and cases. There is slight difference in presence of risk factor like smoking diabetes among the cases and controls; case group had slightly more (although non-significant) diabetic and smokers than controls. Whether this could have caused higher occurrence of acute coronary syndrome is debatable, lipoprotein(a) being just innocent bystander. However it is described that all these coronary risk factors do not affect the level of Lpa level. Because of small sample size, difference in the Lpa level among ethnic groups could not be analysed as done in other studies. ${ }^{9-17}$

Some studies had controversial reports that statin increases the Lpa level and aspirin decreases the level. ${ }^{18}$ In our study all patients were on aspirin and atorvastatin. This would probably balance the net effect on Lpa if any. And the recent studies ${ }^{19-22}$ show that nicotinic acid in various combinations lower Lpa and is beneficial for coronary artery disease.
These days, many young patients are found to have coronary artery disease. Other than smoking, risk factors are not usually seen in such patients. Higher prevalence of Lpa could not be selectively demonstrated among young patients probably because of small sample size in our study, which may be regarded as the drawback of the study. Niacin has multiple effects like anti-oxidant anti-inflammatory and lipid lowering effect as well as Lpa lowering effect. So what caused reduction in clinical effect would not be very easy to determine.

A distinctly elevated Lpa level is an important risk factor for an early-onset and badly progressive arteriosclerosis. ${ }^{23}$ Lowering serum Lpa level in these patients may control the progression of coronary artery disease. The NAUTILUS ${ }^{24,25}$ study demonstrated the beneficial efficacy and tolerability profiles of sustained release nicotinic acid in a usual-care setting. The most common side-effect of niacin is flushing, which infrequently causes treatment discontinuation and which usually subsides over continued treatment. These studies have used nicotinic acid in high dose 1.5 gram to 2 gram for upto 20 weeks. This study has targeted to avoid those sideeffects by using low dose. The ARBITER $2^{26}$ study showed $1-2$ years of treatment with niacin plus a statin induced regression of atherosclerosis in patients with coronary artery disease. The mechanism of the benefit achieved by niacin has not been explained in many studies. However in another study ${ }^{27}$ it has been suggested that the mechanism is through improvement in endothelial function and that improvement is present in patients with low High density lipoprotein cholesterol (HDL-C), but not with normal HDL-C.

The limitation of the study is the lack of clinical correlation with the events after the treatment. Because of logistic reasons this could not be carried out. The technical difficulties in assessing the Lpa level are recognized however this has been attempted sincerely by proper and regular standard methods.

\section{CONCLUSION}

The serum level of Lpa is found to be higher among coronary artery disease patients and appears to be a strong risk factor for coronary artery disease in our population also. The serum level of Lpa was not found to be significantly different in different types of acute coronary syndrome - STEMI, NSTEMI and unstable angina. Administration of low dose of nicotinic acid reduced the serum Lpa level significantly in patients of acute coronary syndrome and was well tolerated.

\section{ACKNOWLEDGEMENT}

The logistic help and advices given by Prof David Colquhoun MD (Prince Royal Albert Hospital Australia) is appreciated. Without his help this study would not have been successful. 


\section{REFERENCES}

1. Cantin B, Gagnon F, Moorjani S. Is Lipoprotein (a) an independent Risk factor for Ischemic Heart Disease in Men? The Quebec Cardiovascular Study. J Am Coll Cardiol 1998;31:519-25.

2. Eckerdstein A, Schultz $H$, Cullen $P$, et al. Lipoprotein (a) Further Increases the risk of coronary Events in Men with High Global Cardiovascular Risk. J Am Coll Cardiol 2001;37:434-9.

3. Luc G, Bard JM, Arveiler D, et al. Lipoprotein (a) as a predictor of coronary heart disease: the PRIME Study. Atherosclerosis 2002;163:377-84.

4. Bhatnagar D. The metabolic basis of increased coronary risk attributed to the people from South Indian subcontinent. Curr Science 1998; 74: 1087-94.

5. Kostner KM, Kostner GM. Lipoprotein (a): Still an enigma? Curr Opin Lipidol 2002; 13: 391-6.

6. Teramoto T, Yamada N, Shimano H et al. Dose-dependent effect of niceritrol on plasma lipoprotein-a. Scand J Clin Lab Invest 1996;56:359-65.

7. Kashyap ML. Long-term safety and efficacy of a once daily niacin/lovastatin formulation for patients with dyslipidaemia. Am J cardiol 2002;89:672-8.

8. Angelin B. Therapy for lowering $L p(a)$ levels. Curr Opin Lipidol 1997;8: 337-41.

9. Nakanishi M, Rai SK, Hirai K, et al. Serum lipid profile in adult Nepalese of different ethnic groups. J Inst Medicine 1997;19:98-104.

10. The Lipid Research Clinics Program Epidemiology Committee. Plasma lipid distributions in selected North American populations: The lipid research clinics program prevalence study. Circulation 1979;60;427-39.

11. Zaraihan S, Azman AB, Tariq AR. Radical differences in the fasting lipid profile of healthy Malaysians. Med J Malaysia 1994;49:355-63.

12. Saha N. Serum high density lipoprotein cholesterol apoliprotein Al, All and B levels in Singapore ethnic groups. Atherosclerosis 1987;68:117-21.

13. Pacy PJ, Dogson PM, Kubicki A, et al. Differences in lipid and lipoprotein levels in white, black and Asia noninsulindependent type 2 diabetes with hypertension. Diabetes Res 1987;4:187-93.

14. Rubinstein A, Landau E, Goldbourt U, et al. Lipids and lipoproteins in new immigrant Ethiopian Jews in Israel. Am J Epidemiol 1998;128:153-64.

15. Ofler'ev AM, Volozh OIPolesslii VA, Sokolova MA, et al. Characteristics of the plasma lipid profile in the male population of Tahinn aged 20-54years. Cor Vasaq 1990;32:441-7.
16. Forsdahl A, Salmi H. A follow-up based on the cardiovascular study done in the country of Finnmark in 1987. Tidsskrr Nor Laegeforen 1990;110;2341-4.

17. Koukkou E, Watts GF, Mazurkiewicz J, et al. Ethnic differences in lipid and lipoprotein metabolism in pregnant women of African and Caucasiam origin. J Clin Pathol 1994;47:1105-7.

18. Akaike M, Azuma $H$, Kagawa A, et al. Effect of aspirin treatment on serum concentrations of lipoprotein(a) in patients with atherosclerotic diseases. Clin Chem 2002;48:1454-9.

19. Vittone $F$, Chait A, Morse JS, et al. Niacin plus simvastatin reduces coronary stenosis progression among patients with metabolic syndrome despite a modest increase in insulin resistance: A subgroup analysis of the HDLAtherosclerosis Treatment Study (HATS). J Clin Lipidol 2007;1:203-10.

20. Bays H. Safety of niacin and simvastatin combination therapy. Am J Cardiol. 2008; 17; 3-8.

21. Davidson $\mathrm{MH}$, Ballantyne CM. Niacin therapy: an evolving paradigm for the management of mixed dyslipidemia and low high-density lipoprotein cholesterol Introduction. Am J Cardiol 2008; 17:1B-2B.

22. Leithäuser B, Gerk $U$, Mrowietz $C$ et al. Influence of xantinole nicotinic acid on cutaneous microcirculation in patients with coronary artery disease and hyperlipoproteinemia. Clin Hemorheol Microcirc 2008;39:287-92.

23. Fischer $\mathrm{S}$, Julius $\mathrm{U}$, Bergmann $\mathrm{S}$. Severe progression of coronary heart disease in a patient with elevated lipoprotein (a) level in spite of optimal LDL-C decrease. Herz 2007 ; 32: 578-82.

24. Kassner $U$, Hostalek $U$, Steinhagen-Thiessen $E$. Prolonged-release nicotinic acid for the management of dyslipidemia: an update including results from the NAUTILUS study. Vasc Health Risk Manag 2007;3:46779.

25. Sanyal S, Karas RH, Kuvin JT. Present-day uses of niacin: effects on lipid and non-lipid parameters. Expert Opin Pharmacother 2007; 8:1711-7.

26. Taylor AJ, Sullenberger LE, Lee HJ, et al. Arterial biology for the investigation of the treatment effects of reducing cholesterol (ARBITER) 2: a double blind, placebo controlled study of extended-release niacin on atherosclerosis progression in secondary prevention patients treated with statin. Circulation 2004;110:3512-7.

27. Warnholtz A, Wild P, Ostad MA, et al. Effects of oral niacin on endothelial dysfunction in patients with coronary artery disease: Results of the randomized, doubleblind, placebo-controlled INEF study. Atherosclerosis 2009;204:216-21. 